\title{
Beautiful, self-absorbed, and shallow: people of color perceive White women as an ethnically marked category
}

\author{
Terri D. Conley \\ Department of Psychology, University of Michigan
}

\begin{abstract}
Correspondence concerning this article should be addressed to Terri D. Conley, Department of Psychology, University of Michigan, 1012 East Hall, 530 Church Street, Ann Arbor, Ml 48103, USA. E-mail: conleyt@umich.edu
\end{abstract}

doi: 10.1111/j.1559-1816.2012.00980.x

\begin{abstract}
Non-Whites' stereotypes of White women were examined, comparing three perspectives: (1) White women are perceived similarly to ethnically "generic" stereotypes of women; (2) stereotypes are opposite of stereotypes of participants' own ethnic group; and (3) stereotypes are derived from media images of White women. In Study 1, participants listed stereotypes of White women in an open-ended fashion. In Study 2, those stereotypes were developed into a close-ended questionnaire, completed by a second set of participants. White women were perceived as attractive, blonde, ditsy, shallow, privileged, sexually available, and appearance focused. We concluded that White women are ethnically marked. Stereotypes of White women are consistent with media images of White women.
\end{abstract}

White women often think of their ethnic identity as generic and think of themselves as "unraced" (Frankenburg, 1993), but do people of color believe White women are race absent or race neutral? In the current research, we identified stereotypes that several ethnic groups hold about White women, examined ethnic and gender differences in those perceptions, and considered the origins of these images.

\section{White women: marked and unmarked}

People of color are "marked" within contemporary U.S. society, whereas White (presumptively male) people are perceived to be "unmarked" (Brekhus, 1998; Damarin, 2000; Goffman, 1963). People of color are perceived as lower status to Whites (Sidanius \& Pratto, 1999). This means that people of color in our society are subjected to a unique set of challenges in a White-dominant society, including prejudice, discrimination, stereotyping of their group, and/or invisibility. Although we in no way dispute the clear fact that people of color are at a disadvantage in our society relative to Whites in a multitude of contexts, we question whether White women are ethnically unmarked. That is, people of color may attribute unique and unflattering meaning to the category of White women. In summary, dominant groups may belong to marked categories (as perceived by marginalized groups) as well.

Marked classes of individuals stand out; that is, they are not generic or universal, but are perceived to have distinguishing and often negative features or qualities (see Brekhus, 1998).
The position of White women in society is complex: They might be considered "marked" in terms of gender but "unmarked" in terms of race. However, if stereotypes about White women are unflattering and peculiar to their ethnic category, we argue that White women are ethnically marked. The content of these stereotypes, of course, is likely less socially troublesome than those ascribed to members of marginalized ethnic groups. For example, to the extent that stereotypes address groups' global competence, they may be especially damaging-but stereotypes of incompetence are not typically attached to dominant groups (see Cuddy, Fiske, \& Glick, 2007; Steele, 1997). The exploration of whether White women are ethnically marked is a central motivation for examining stereotypes about White women.

\section{Why are perceptions of Whites so infrequently studied?}

Very little research has examined perceptions of Whites (Shelton, 2000; Shelton \& Richeson, 2006) and even less has assessed perceptions of White women specifically. Within the field of psychology, we know a great deal about how Whites conceptualize African Americans (and to a lesser extent, other non-White ethnic groups and gay people) but precious little about how members of other ethnic groups perceive Whites. Yet, marginalized group members undoubtedly bring their own motivations and expectations to the intergroup dynamic (see Conley, Calhoun, Evett, \& Devine, 2001). 
We suggest that the pervasive focus on Whites' attitudes toward people of color may have emerged as a well-meaning attempt to put the responsibility for prejudice on the major perpetrators of prejudice (i.e., the dominant group; Shelton, 2000). Indeed, examining stereotypes about Whites could be interpreted as justifying the prejudice that people of color have experienced at the hands of Whites.

Alternatively, we suggest that White dominance may have implicitly affected the research inquiries. As Sue (2004) noted, most Whites fail to realize the ways in which "their Whiteness intrudes upon persons of color" (p. 763); we suggest that a failure to understand Whiteness could translate into the supposition that targets of prejudice are simply reacting to Whites, rather than bringing their own motivations and concerns to the intergroup dynamic.

Recently, researchers have called for more attention to the "unmarked category," which, in the case of ethnicity in the United States, would be White people (Brekhus, 1998). The current research addressed the unmarked category of Whiteness in the United States.

\section{Perspectives on the content of stereotypes about White women}

We considered three possible perspectives on the content of stereotypes about White women: that the content of White female stereotypes would be substantially similar to that of general stereotypes of women; that stereotypes would be opposite of those of the groups generating the stereotypes (as proposed by self-categorization theory); and that the stereotypes will closely resemble media images of White women.

\section{Stereotypes of White women as stereotypes of ethnically generic women}

Given that stereotypes about (presumably ethnically generic) women include emotionality, kindness, incompetence, and talkativeness (e.g., Cowan \& Warren, 1994; Prentice \& Carranza, 2002), we suspect that most (predominantly White) participants in gender stereotyping studies think of White women when they think of the category "woman." In one of the few studies actually addressing White women specifically, Landrine presented undergraduates (more than 90\% of White women) with a list of adjectives and had participants indicate the extent to which society associates these stereotypes with White (or Black) women. Landrine (1985) concluded that stereotypes of White women are fundamentally similar to stereotypes of women more generally. Therefore, among Whites, stereotypes about White women may be largely redundant with stereotypes of women. It is unclear whether people of color would share these perceptions. Moreover, Landrine's sample utilized a preexisting set of traits, rather than asking participants to generate stereotypes.
It is possible that participants, not accustomed to thinking of White women as having any special characteristics, defaulted to other accessible stereotypes (i.e., gender stereotypes) when asked to recognize stereotypes about White women among other characteristics.

\section{Stereotypes of White women as opposite of stereotypes of your own group}

Self-categorization theory proposes that stereotypes are about group differences (e.g., Mlicki \& Ellemers, 1998). Therefore, stereotypes between groups, based on the selfcategorization perspective, should be opposite in content. This theory predicts that stereotypes of White women will be opposite of the stereotypes about women in the participant's own ethnic group. That is, African Americans will have stereotypes of Whites that are opposite of the stereotypes about African Americans, Asian Americans will have stereotypes of Whites opposite of the stereotypes of Asian Americans, etc. According to self-categorization theory, we would expect meaningful ethnic differences in perceptions of the content of White women's stereotypes, because the content of stereotypes about different ethnic groups is different (that is, stereotypes about Latinas/os are different from stereotypes about African Americans, which are also different from stereotypes about Asian Americans). Because Whites hold different stereotypical images of different ethnic groups, people of color should correspondingly have different images of Whites based on self-categorization theory.

\section{Stereotypes of White women reflect media images}

Finally, we suggest that White women are uniquely singled out in the visual media. In contemporary U.S. society, we likely have more information about what idealized White women look like than any other ethnic $\times$ gender group combination (see Conley \& Ramsey, 2011). The media images directed toward women (and usually portraying White women) are largely focused on attractiveness and weight regulation (Evans, Rutberg, Sather, \& Turner, 1991; Malkin, Wornian, \& Chrisler, 1999; Snow \& Harris, 1986) and feature unusually thin, disproportionately blonde characters or models (Davis, 1990; Elasmar, Hasegawa, \& Brain, 1999; Rich \& Cash, 1993). Women are portrayed in dependent and submissive roles (Peirce, 1993; Russo, Feller, \& DeLeon, 1982) as sexually available (Davis, 1990; Seidman, 1992; Signorielli, McLeod, \& Healy, 1994), very fit (Signorielli et al., 1994), and universally young and attractive (Davis, 1990; Downs \& Harrison, 1985). We would also argue that social class is another aspect of the media image of White women, given that White women are portrayed as having the resources to care excessively about their appearance. As Davis (1990) notes regard- 
ing television portrayals of women, the image that the media present is "of the young, attractive, and sexy female who is more ornamental ... than functional," (p. 311).

We also argue that the U.S. population receives more prescriptive information about White women's appearance than any other gender $\times$ ethnic group combination. Whereas stereotypes of other groups may be defined to a greater extent by shared perceptions among one's own group (i.e., what an individual and her family, friends, and coworkers believe are the characteristics of a given group), we suggest that White women are especially likely to be defined through the media. Therefore, based on the media perspective, we might expect stereotypes of White women to reflect media images.

\section{Previous research on stereotypes of Whites}

We are aware of two additional research projects that have specifically examined stereotypes of Whites. First, Conley, Rabinowitz, and Rabow (2010) addressed stereotypes that people of color hold about White men. That research demonstrated that White men are viewed in multifaceted ways, suggesting the existence of subtypes: the frat boy, the greedy businessman, and the nice guy. Are images of White women similarly multifaceted, suggesting different subtypes of the larger category of White women? Or are they unidimensional? Self-categorization would suggest a greater number of differences-because the stereotypes would be opposite of each ethnic group's stereotypes (e.g., stereotypes about White women would be opposite of one set of stereotypes for Asian Americans, another for Latinas/os, and another for African Americans). By contrast, to the extent that stereotypes about White women are derived from ethnically generic female stereotypes or from media images of White women, we would expect fairly unidimensional perceptions of White women.

Second, Niemann, Jennings, Rozelle, Baxter, and Sullivan (1994) elicited open-ended perceptions of eight ethnic $\times$ gender groups, including White women. Participants were from a variety of ethnic backgrounds, although still largely White. Niemann et al. (1994) found that White women were perceived as attractive, intelligent, pleasant/friendly, egotistical, blonde/light haired, and sociable/socially active. Interestingly, some of the stereotypes (i.e., pleasant/friendly and sociable/socially active) are consistent with the general stereotype of ethnicity-unspecified women, but others (e.g., intelligent, egotistical) run counter to cultural stereotypes about women. Therefore, support for the perspective suggesting that White women are perceived as "generic" women is mixed.

We do not clearly sense whether stereotypes of White women are derived from media images in Niemann et al.'s stereotypes; some do appear to be consistent with media images (like blonde and attractive), whereas others (intelli- gent) do not. However, the coding categories from Niemann et al. may not have been sufficiently narrow to consider different groups' perceptions of White women. Similar problems emerge in interpreting stereotypes of White women within the framework of self-categorization theory. Because the stereotypes were not examined separately by ethnic group, it is difficult to determine if specific stereotypes that one ethnic group holds about White women are opposite of stereotypes about that ethnic group. In the current research, we considered stereotypes about White women more thoroughly, elucidating more specific categories of stereotypes and making comparisons between ethnic groups. This will aid us in addressing possible origins of White female stereotypes.

\section{Gender differences in perceptions of White women}

Interestingly, in the aforementioned research on perceptions of White men (Conley, Rabinowitz, \& Rabow, 2010) no perceiver gender differences emerged. However, because White women as a group are subordinated by men in contemporary U.S. culture, we might find that women of color are relatively charitable in their perceptions of White women. That is, women of color might relate to White women because of their gender and have more sympathetic perceptions of White women than men of color do. However, members of marginalized groups clearly are not always unified in support for one another, despite often sharing common experiences of oppression (Jost \& Banaji, 1994). To the extent that the relationship between women of color and White women is marked by tension rather than unity, we might expect women participants to perceive White women especially negatively.

We have also raised the possibility that media images are responsible for images of White women. Women may be better attuned to these media stereotypes. They may be more aware of how, as women of color, they by definition cannot live up to the idealized White female media images. We suggest that this might cause women of color to more strongly endorse stereotypes about White women that are consistent with media images of White women (i.e., stereotypes focused on appearance and beauty).

\section{The current research}

In the current research, we recruited samples of three ethnic groups, African Americans, Asian Americans, and Latinas/os, to make assessments of differences between the groups' stereotypes about White women. Like Niemann et al. (1994), we took a multimethod approach to understanding stereotypes of White women. Specifically, we considered stereotypes elicited both through a free-listing, open-ended approach and through a close-ended questionnaire. 


\section{Study 1}

In Study 1, we elicited stereotypes of White women from three ethnic groups, African Americans, Asian Americans, and Latinas/os, via open-ended responses. We were interested in assessing the content of stereotypes about White women and determining if this content varied by gender or ethnicity.

\section{Method}

\section{Participants and procedure}

The sample ( $n=110)$ was $64 \%$ female and consisted of three ethnicities: African Americans (16\%), Latinas/os (33\%), and Asian Americans (52\%). The average age was 23 years $(S D=5.15)$. Research assistants approached individuals in a large, urban, West coast college campus and asked them to complete the questionnaires. College students were not specifically targeted. Although we presume that most participants were students, not all were students (or at least traditionally aged students) based on the age mean, which was 23 (i.e., traditional college students graduate at 21 or 22).

Participants described, in open-ended responses, the stereotypes that people have about White women. Participants returned the questionnaires to researchers in sealed envelopes.

\section{Materials}

Early pilot testing indicated that the topic "White stereotypes" was highly reactive. Therefore, instead of asking about participants' own stereotypes, we instead asked about other people's stereotypes (cf. Fiske et al., 2002) in both Study 1 and Study 2. Personally held stereotypes and cultural stereotypes are highly correlated (Gordijn, Koomen, \& Stapel, 2001; Krueger, 1996), indicating that this assessment method yields veridical stereotypes.

The questionnaire indicated that we were interested in stereotypes about White women and participants were asked to "list the traits, qualities and behaviors that people associate with White women." We explained that they should list all aspects of the stereotype, even if they did not agree with the belief. We urged participants to list both positive and negative qualities of White women.

\section{Coding}

We coded the stereotypes utilizing techniques outlined by Backstrom and Hursh-Cesar (1981). First two coders independently identified themes in the responses. They created general categories through consensus (i.e., they jointly determined how broad/narrow each stereotype category should be). These categories formed the coding scheme. Both coders reviewed each respondent's list of stereotypes and indicated whether each stereotype theme was present or absent in each
Table 1 Full Name of Coding Category and Associated Nickname, Study 1

\begin{tabular}{ll}
\hline Nickname & Full category \\
\hline Ditsy & Unintelligent/airheads/ditsy \\
Beautiful & Beautiful/attractive/standard of beauty \\
Blonde & Blonde \\
Blue eyes & Blue eyes \\
Dieting & Concerned about appearance/dieting all the time \\
Materialistic & Love to shop/shopaholics/materialistic \\
Sexually easy & Sexually easy \\
Rich & Rich \\
Tall & Tall \\
Trashy & White trash/trashy \\
Health-conscious & Health-conscious/always going to the gym \\
Immoral & Immoral/not to be trusted/gold digger \\
Conceited & Conceited/stuck-up/snobbish \\
Shallow & Fake/shallow/plastic \\
Bitchy & Bitchy \\
Feminists & Feminists/believe in equality \\
Independent & Career-oriented/independent \\
Big breasts & Big breasts \\
Pale skin & Pale skin \\
Unclean & Unclean \\
Racist & Ethnocentric/racist/uninformed about other cultures \\
Loud & Loud/outspoken \\
Dependent & Dependent \\
Date interracially & Date interracially \\
Cater to men & Cater to men \\
Privileged & Advantaged/privileged/have more opportunities \\
& than women of other ethnicities \\
Uneducated & Uneducated \\
\hline & \\
\hline
\end{tabular}

response. Inter-rater agreement was $93 \%$, with discrepancies resolved by a third coder. The coding categories and nicknames (which will be used to refer to the categories) are included in Table 1.

\section{Results and discussion}

We first considered the most common stereotypes of White women and then examined ethnic and gender differences in listing the stereotypes.

The most common stereotypes listed by each ethnic group are included in Table 1. Four stereotypes were among the top ten stereotypes listed by all three ethnic groups: dumb, snobby, sexually easy, and beautiful. In addition, blonde, dieting/appearance-focused, racist, shopaholics, untrustworthy/ immoral, and career-oriented were listed among the top ten stereotypes for two out of the three groups. These stereotypes do not closely resemble stereotypes of women in general (cf. Prentice \& Carranza, 2002), suggesting that White women belong to an ethnically marked category. Many of these stereotypes do correspond to media images of White women as ornamental and sexually available. 
Table 2 Most Frequently Listed Stereotypes by Ethnic Group, Study 1

\begin{tabular}{lllll}
\hline Asian Americans & \% reporting & African Americans & \% reporting & Latina/os reporting \\
\hline Ditsy & 35 & Dependent & 47 & Ditsy \\
Conceited & 33 & Conceited & 35 & Conceited \\
Blonde & 29 & Date interracially & 29 & Racist \\
Sexually easy & 26 & Ditsy & 29 & Blonde \\
Beautiful & 23 & Immoral & 24 & Sexually easy \\
Dieting & 18 & Beautiful & 24 & Shopaholics \\
Tall & 18 & Racist & 24 & 28 \\
Feminists & 16 & Independent & 18 & Independent \\
Loud & 14 & Sexually easy & 18 & Beautiful \\
Trashy & 14 & Rich & 18 & Rich \\
Shopaholics & 14 & Dieting & 18 & 18 \\
\hline
\end{tabular}

To examine the self-categorization claim that stereotypes address how groups differ, we considered differences in the top-ranked stereotypes by ethnic group. Contrary to the claim of self-categorization theory that stereotypes are about group differences, the stereotypes that these three ethnic groups listed were on the whole consistent across the groups (see Table 2). However, there were a few exceptions that provided some support for self-categorization theory. Asian Americans had the greatest number of stereotypes in their top ten that were unique to their own group (i.e., that were not among the other groups' frequently listed stereotypes). Specifically, Asian Americans listed loud, trashy, feminists, and tall within their top ten stereotypes, whereas neither of the other groups did. Consistent with self-categorization theory, these stereotypes seem to be opposite of prominent stereotypes of Asian women as quiet, well-mannered, passive, shy (i.e., not outspoken, like feminists are often perceived to be), and short (Niemann et al., 1994).

African Americans' most frequently listed stereotype, of White women as dependent/weak, was not listed by other groups. Given stereotypes of Black women as strong (e.g., West, 2008), this unique stereotype is consistent with self-categorization theory's predictions of White women's stereotypes being opposite of the stereotyping group (i.e., dependent or weak). Latinas/os' stereotypes were largely consistent with those of the other groups and all of their top stereotypes were listed by other groups. In this first study, self-categorization's premise that stereotypes are about group differences was most applicable to stereotypes held by Asian Americans.

Finally, we were interested in whether the stereotypes generated by participants in Niemann et al. (1994) corresponded to those we found. We considered the most frequently mentioned stereotypes from Niemann et al.'s study: sociable/socially active, intelligent, pleasant/friendly, blonde, attractive, and egotistical. Interestingly, the positive traits of sociable/socially active, intelligent, and pleasant/friendly did not emerge in our list of stereotypes. Only the physical descriptors (blonde, attractive) and the negative item egotistical emerged in the current stereotype list. Perhaps the more positive perceptions of White women in Niemann et al. (1994) were a result of the large number of White participants completing the questionnaires.

\section{Ethnic differences in stereotypes about White women}

Next we considered ethnic differences in the likelihood of listing stereotype categories (i.e., the frequency with which each of the stereotypes was listed, by ethnic group). We first conducted chi-square analyses to detect differences among the three ethnic groups in listing the stereotype. Significant differences emerged in listing the stereotypes of blonde: $\chi^{2}(2)=6.53, \quad p<.038 ; \quad$ racist: $\quad \chi^{2}(2)=10.67, \quad p<.005 ;$ dependent/weak: $\chi^{2}(2)=26.18, p<.0005$; and date interracially: $\chi^{2}(2)=13.32, p<.008$.

To determine which ethnic comparisons were driving these significant differences, we conducted pairwise comparisons via chi-square tests comparing each pair of groups. African Americans were more likely than Asian Americans, $\chi^{2}(1)=10.26, p<.001$, or Latinas/os, $\chi^{2}(1)=6.37, p<.012$, to list the stereotype date interracially. African Americans were also more likely than Latinas/os, $\chi^{2}(1)=7.94, p<.005$, or Asian Americans, $\chi^{2}(1)=25.16, p<.0005$, to list the stereotype dependent/weak. Conversely, Latinas/os, $\chi^{2}(1)=4.84$, $p<.028$, and Asian Americans, $\chi^{2}(1)=6.58, p<.01$, were more likely to list the stereotype blonde than were African Americans. Asian Americans and Latinas/os did not differ in the frequency of listing any of these stereotypes.

The only other significant difference was that Latinas/os were more likely to list the stereotype racist than were Asian Americans, $\chi^{2}(1)=10.79, p<.001$. African Americans and Latinas/os did not differ in the frequency of listing this stereotype. 


\section{Gender comparisons}

Across all the stereotype categories, date interracially was the only item that yielded a gender difference, $\chi^{2}(1)=5.67$, $p<.023$. Men were more likely to mention this stereotype $(15 \%)$ than women were $(3 \%)$. To the extent that White women are sexually objectified in the media, it may be more relevant to the men in our sample (who were likely predominantly heterosexual) than to the women, whether White women would be attracted to men of color.

\section{Summary}

Open-ended stereotypes of White women were focused on their appearance and self-absorption. We saw some evidence for the self-categorization perspective in explaining the content of the stereotypes; some of the stereotypes generated about White women appeared to be opposite of perceptions of women of color (e.g., White women perceived as tall by Asian American participants and weak by African American participants). We also observed that many of the stereotypes of White women are similar to media images of White women: blonde, beautiful, sexually easy, and unintelligent.

This study is a first step in understanding stereotypes of White women. However, participants may have had difficulty in generating stereotypes about White women because they are not accustomed to thinking about stereotypes of dominant groups. Therefore, they might have been essentially creating stereotypes that they had not previously considered in response to the questionnaire (Niemann et al., 1994). Moreover, Study 1 was relatively small; small samples tend to generate a higher number of idiosyncratic responses (Niemann et al., 1994). To further validate these stereotypes of White women as generally held cultural stereotypes, we conducted a second study in which participants responded to stereotypes about White women in a close-ended fashion.

\section{Study 2}

In Study 2, the stereotypes that participants generated in Study 1 were developed into a close-ended questionnaire. We presented this questionnaire to African Americans, Asian Americans, and Latinos/as. This study helped us validate the stereotypes generated in Study 1 using a more standardized assessment technique and a larger sample.

\section{Method}

\section{Participants}

Participants $(n=424,64 \%$ female, mean age $=24$ years, $S D=7.7$ ) were recruited in public places of large urban campuses. The ethnic groups included African Americans (23\%), Asian Americans (56\%), and Latinas/os (21\%).

\section{Measures}

Categories from Study 1 were adapted into items that participants responded to on 7-point scales, where higher numbers indicate greater awareness of the stereotypes. To develop the stereotype coding categories from Study 1 into manageable items, we made a few modifications to reduce confusion among the participants. Specifically, when the subcategories in a given category were not clearly synonymous, we divided those categories into separate items. For example, the category concerned about appearance/dieting all the time was divided into two items: concerned about appearance and dieting all the time. Similarly, the category love to shop/ shopaholics/materialistic was divided into two items: love to shop or shopaholics and materialistic. In general, though, we tried to maintain the integrity of the categories we developed for the coding system. The exact list of items is provided in Table 3.

We included two stereotype foils (speak with an accent and are good at math and science) that were explicitly not associated with White females. This procedure ensured that we would have a way to distinguish stereotypes of White women from non-stereotypes. If participants simply endorsed every stereotype strongly, it would indicate a lack of easily discernible stereotype of White women.

We also gave participants space to describe additional stereotypes (i.e., those not included in the questionnaire). Upon reviewing these open-ended responses, we found that the stereotypes participants listed there were redundant with categories already included in the questionnaire (e.g., bimbo would be listed as an additional stereotype, but the similar terms ditsy and sexually easy were already included in the questionnaire) or idiosyncratic (i.e., only listed by one participant). We concluded that the items on our questionnaire were a reasonable representation of stereotypes of White women.

\section{Procedure}

The procedure was the same as Study 1 .

\section{Results and discussion}

First, we examined the responses to determine which stereotypes of White women were well recognized by the three ethnic groups. These items were factor analyzed and the underlying dimensions were developed into scales. We then examined ethnic and gender differences in the perceptions of White women utilizing two-way analyses of variance (ANOVAs).

\section{Stereotype recognition}

We reasoned that stereotypes should be at least slightly recognized by at least one group to be considered a true stereotype. 
Table 3 Full Name of Item and Associated Nickname, Study 2

\begin{tabular}{ll}
\hline Nickname & Full text of item \\
\hline Ditsy & Are unintelligent, airheads, ditsy \\
Beautiful & Are beautiful or are the standards of beauty \\
& in this society \\
Blonde & Are blonde \\
Materialistic & Are materialistic \\
Appearance-focused & Are concerned about their appearance \\
Shopaholics & Love to shop, are "shopaholics" \\
Sexually easy & Are sexually easy \\
Dieting & Are dieting all the time \\
Trashy & Are trashy/"white trash" \\
Health-conscious & Spend a lot of time working out or going to \\
& the gym \\
Immoral & Are immoral or cannot be trusted \\
Conceited & Are conceited, stuck-up, snobbish \\
Shallow & Are shallow or fake \\
Bitchy & Are "bitchy" \\
Feminists & Are feminists \\
Independent & Are independent, career-oriented \\
Big breasts & Have large breasts \\
Pale skin & Have pale skin \\
Unclean & Are dirty, not clean \\
Racist & Are ethnocentric, racist, or ignorant about \\
& non-White cultures \\
Dependent & Are dependent or weak \\
Date interracially & Like to date men of other ethnicities \\
Cater to men & Cater to men, are submissive to men \\
Privileged & Are privileged or have more opportunities in \\
this culture \\
Uneducated & Are uneducated \\
Tall & Are tall \\
Blue eyes & Have blue eyes \\
Rich & Are rich \\
Loud & Are outspoken or loud \\
Speak with an & Speak with an accent or don't speak English \\
\hline
\end{tabular}

Therefore, we retained stereotypes that were rated at least slightly higher than the midpoint of the scale on average (i.e., above 4.25 on the 7-point scale) for at least one of the groups; these are listed in Table 4. Notably, the stereotype foils were the lowest rated items for each of the ethnic groups, confirming that the stereotypes are recognizable and not merely primed by our questionnaire.

As in Study 1, stereotypes about White women are quite similar between ethnic groups and seem consistent with media images. The stereotypes of pale skin, blonde, blue eyes, beautiful, privileged, dieting, materialistic, shopaholics, appearance-focused, and sexually easy were rated above 4.25 for all groups, whereas the stereotypes shallow, conceited, ditsy, bitchy, and health-conscious were listed by two of the three groups. The stereotype privileged is consistent with prior research on perceptions of White men (Conley et al., 2010); however, the remaining stereotypes seem to be unique to White women.

A few group-specific stereotypes emerged. Asian Americans were the only group for which the item large breasts was well recognized. African Americans ranked highly the items cater to men and date interracially. Latinas/os were the only group for whom the stereotype rich was above threshold.

The stereotypes that emerged in the coding scheme in Study 1, but did not resonate with participants in Study 2, included: cater to men, dirty, uneducated, trashy, and feminist. These items suggest the existence of subtypes of White women, i.e., the lower class White woman (Landrine, 1985), and the feminist (Twenge \& Zucker, 1999). Apparently, these subtypes are not a part of the culturally dominant stereotypes of White women that we elicited in this research. It would be interesting to explore these dimensions in future research.

In summary, stereotypes about White women appear to be relatively consistent across these two methods, with a few exceptions. First, the stereotype ditsy was much more prominent in open-ended (Study 1) than in close-ended (Study 2) responses. We suspect that this is a methodological artifact of the coding system: the stereotype blonde was often (but not always) paired with the stereotype dumb (hence, the "dumb blonde"). Because these two items were only sometimes paired, we decoupled them in the coding system. We suspect that the term blonde does convey the stereotype ditsy for many of our respondents. Second, the most frequently listed stereotype of White women listed by African Americans in the free-listing task was dependent/weak. However, this stereotype did not even reach threshold for inclusion in the remaining analyses for Study 2.

Finally, the stereotype privileged was much more prominent in Study 2. Although the concept of privilege was implicit in many of the other stereotypes that emerged in Study 1 (i.e., those addressing consumerism and appearance), it was not explicitly mentioned by many participants. Perhaps the stereotype of privilege is one that was almost too obvious to be mentioned in Study 1 (inasmuch as it was associated with so many of the other more specific stereotypes that participants listed). By contrast, when participants were presented with privilege as a stereotype, they recognized it clearly.

\section{Factor analyses}

Factor structure was analyzed using a principal components factor analysis alternately with varimax rotation and oblique rotation, and confirmed with a maximum likelihood analysis. In addition, the analyses were conducted separately for each ethnic group to assure a mutually recognizable set of factors for all three groups.

Across these analyses, scree plots and weights strongly suggested a two-factor solution. The personal characteristics 
Table 4 Stereotypes Retained for Factor Analyses (Stereotypes That Are Rated Above 4.25 on a 7-Point Scale) by Ethnic Group, Study 2

\begin{tabular}{|c|c|c|c|c|c|}
\hline \multicolumn{2}{|l|}{ Asian Americans } & \multicolumn{2}{|l|}{ African Americans } & \multicolumn{2}{|l|}{ Latinas/os } \\
\hline Beautiful & 5.18 & Privileged & 5.76 & Pale skin & 5.72 \\
\hline Pale skin & 5.11 & Dieting & 5.36 & Appearance-focused & 5.60 \\
\hline Privileged & 4.97 & Shopaholics & 5.31 & Privileged & 5.58 \\
\hline Appearance-focused & 4.72 & Appearance-focused & 5.27 & Blonde & 5.46 \\
\hline Tall & 4.69 & Conceited & 5.07 & Shopaholics & 5.40 \\
\hline Blonde & 4.69 & Materialistic & 5.07 & Beautiful & 5.37 \\
\hline Shopaholics & 4.53 & Sexually easy & 5.06 & Blue eyes & 5.36 \\
\hline Large breasts & 4.50 & Shallow & 5.04 & Materialistic & 5.36 \\
\hline Blue eyes & 4.49 & Date interracially & 4.97 & Conceited & 5.26 \\
\hline Sexually easy & 4.48 & Cater to men & 4.93 & Sexually easy & 5.23 \\
\hline Materialistic & 4.34 & Bitchy & 4.89 & Dieting & 5.06 \\
\hline Dieting & 4.33 & Pale skin & 4.79 & Independent & 4.97 \\
\hline \multirow[t]{7}{*}{ Independent } & 4.29 & Racist & 4.70 & Racist & 4.90 \\
\hline & & Blonde & 4.66 & Shallow & 4.86 \\
\hline & & Ditsy & 4.65 & Bitchy & 4.86 \\
\hline & & Health-conscious & 4.50 & Rich & 4.81 \\
\hline & & Beautiful & 4.35 & Health-conscious & 4.75 \\
\hline & & & & Ditsy & 4.71 \\
\hline & & & & Tall & 4.28 \\
\hline
\end{tabular}

factor $(\alpha=.90)$ included the items: dieting, shopaholics, appearance-focused, conceited, materialistic, privileged, sexually easy, shallow, date interracially, cater to men, bitchy, racist, ditsy, and health-conscious. The appearance factor $(\alpha=.76)$ contained the items: pale skin, blonde, beautiful, tall, blue eyes, and large breasts. These two factors seem to represent different components of a single image of White women. The items independent and rich were retained as single items because they inconsistently loaded on the factors.

\section{Ethnic and gender differences in perceptions of the White female stereotype}

A series of 2 (Gender) $\times 3$ (Ethnic Group) ANOVAs were conducted on the dimensions that emerged from the factor analyses, as well as the single-item measures. Significant ethnic differences emerged in the personal characteristics factor, $F(2,357)=23.92, p<.0005$. Post hoc tests revealed that African Americans $(M=5.05)$ and Latinas/os $(M=5.04)$ recognized this stereotype more readily than Asian Americans $(M=4.20)$. Ethnic differences also emerged on the descriptive appearance factor, $F(2,357)=18.91, p<.0005$. Asian Americans $(M=4.78)$ and Latinas/os $(M=5.01)$ recognized this stereotype to a greater extent than did African Americans $(M=4.04)$. The finding that Asian Americans were less likely to recognize the personal characteristics factor, which also was the most subjectively negative factor, is consistent with research on stereotypes of White men, in which Asian Americans had the most positive images of Whites. Asian Americans' positive perceptions of Whites apparently extend to White women as well. We also found significant differences on the single-item stereotype of independent, $F(2,356)=8.08, p<.0005$. Latinas/os $(M=$ $4.97)$ recognized this stereotype to a greater extent than Asian Americans $(M=4.29)$ or African Americans $(M=3.97)$. Latinas/os also recognized the item rich to a greater extent $(M=4.81)$ than Asian Americans $(M=3.98)$ or African Americans $(M=4.14), F(2,356)=6.56, p<.001$.

In addition, significant main effects of gender emerged for several of the stereotypes. Women participants recognized the personal characteristics factor more $(M=4.50)$ than men $(M=4.13), F(2,357)=23.92, p<.0005$. They also recognized the appearance stereotypes more $(M=4.74)$ than men $(M=4.40), F(2,357)=18.91$, and the item independent to a greater extent $(M=4.50)$ than men did $(M=4.14), F(2$, $357)=8.08, p<.005$. Women appear to be much more cognizant of the stereotype of White women than men are.

Notably, there were no interactions between gender and ethnic group.

\section{Summary}

These findings are generally consistent with those of Study 1 . Once again, these images are most similar to those of media images of White women. We found less evidence for stereotypes as group differences, perhaps because participants were recognizing, rather than generating stereotypes. As in Study 1, the stereotypes of White women did not appear to substantially overlap with general stereotypes of women. We found broader gender differences in Study 2 than in Study 1, suggesting that female participants were more in tune with stereotypes of White women than male participants were. Ethnic differences seem to indicate that Asian Americans have 
somewhat more positive stereotypes than the other two ethnic groups.

\section{General discussion}

In this study, we examined three ethnic groups' stereotypes of White women. Consistent with the statements of Whiteness studies scholars, it does not appear that White women are "race-neutral" to people of color (Frankenburg, 1993; Sue, 2004). In fact, the category of White women is associated with a very particular set of traits; White women are perceived as attractive, but shallow and self-absorbed (i.e., materialistic, focused on their appearance, and shopaholics). Thus, we conclude that White women are ethnically marked, if not among themselves, at least in the eyes of people of color. Although some specific stereotypes resonate more with one ethnic group than another (consistent with the premise that stereotypes are about group differences), for the most part, ethnic groups seem to be in agreement with one another about the predominant image of White women. Across the two studies, we suggest that non-Whites' stereotypes of White women correspond most closely to media images of White women.

\section{Content of White female stereotypes}

We proposed three perspectives addressing how White women are perceived: stereotypes emerging from media images of White women; self-categorization perceptions of difference from White women; and perceptions of White women as "generic" women. We will consider each of these perspectives in turn.

\section{Media as potential determinants of stereotypes}

Our findings suggest that stereotypes of White women resemble our idealized images of models and movie stars. Indeed, if the prevalence of advertising regarding plastic surgery and dieting is any indication, this may be an ideal that many White women themselves hold, especially given that exposure to media images is associated with dissatisfaction with one's body among White women (Grabe, Ward, \& Hyde, 2008). White women may well be the most frequently objectified figures in American media. Therefore, stereotypes about White women may be relatively less related to structural variables such as frequency of contact and perceived competition than other groups. Testing this hypothesis would be an intriguing avenue to demonstrate how intersections between ethnicity and gender can inform basic stereotyping and prejudice research.

To further assess whether the stereotypes are formed in response to media images, researchers need to determine if these effects are moderated by exposure to media. If those involved more closely with media on a daily basis are more apt to recognize these stereotypes, it would provide stronger support for the hypothesis that media are responsible for stereotypes of White women.

\section{Self-categorization}

We found some evidence of the self-categorization account of stereotypes as group differences. For example, Asian Americans, who are stereotyped as short, quiet, and passive (Niemann et al., 1994), stereotyped White women as tall, loud, and feminists. African Americans, who are stereotyped as independent and strong (West, 2008), stereotyped White women as dependent and weak. This perspective was better supported in the free-listing study (Study 1) than in the closeended study (Study 2) and better supported among Asian Americans than the other Latinas/os or African Americans. We suggest that people may be more likely to generate opposite stereotypes when they are generating stereotypes about a group that they have not been accustomed to stereotyping (i.e., when the stereotypes are less cognitively accessible). We can only speculate as to why Asian Americans were somewhat more likely to list or recognize stereotypes that are opposite of stereotypes of their own group. Perhaps Asian Americans' interactions with Whites are not as broadly scrutinized in our society, given Asian Americans relatively higher status in U.S. society relative to African Americans or Latinas/os (Sidanius \& Pratto, 1999). This might mean that Asian Americans have thought less about White stereotypes than other ethnic groups have, because the intergroup dynamic may not be made as salient to Asian Americans as it is to Latinas/os and African Americans. As a result, they may be more likely to look to stereotypes of their own group to determine stereotypes about Whites.

\section{Race-neutrality stereotypes about White women}

Prior research investigating stereotypes of White women among Whites suggested that these stereotypes are race neutral (i.e., similar to stereotypes of women in general; Landrine, 1985). Across the two studies, we found minimal evidence for this perspective. The fact that White women are perceived differently than women in general is consistent with our hypothesis that White women are ethnically marked.

\section{Stereotypes of White women vs. White men: complexity and ethnic differences}

Previous research (Conley et al., 2010) revealed that dimensions of the White male stereotype were multifaceted, suggesting the existence of subtypes. White men were grouped into subtypes: the greedy businessman, the frat boy, and the nice guy. By contrast, it appears that our participants have one specific image in their head when they consider White 
women, closely related to media images of White women: beautiful, but self-absorbed and shallow. The fact that White women were perceived largely in terms of their appearance is of note. This is consistent with how all women are typically perceived; that is, women are judged by how attractive they are to others (cf. Fredrickson \& Roberts, 1997).

African Americans were less likely to recognize appearance-related stereotypes in both studies. Perhaps because African Americans are more likely to reject idealized images of White women as the standard of beauty (cf. Makkar \& Strube, 1995), the stereotypes related to White women's appearance did not resonate with them. Asian Americans, consistent with previous research on White men, perceived White women somewhat more positively (or less negatively) than African Americans and (to a lesser extent) Latinos/as. One interpretation of this finding is that Asian Americans interact with Whites more often than Latinos/as or African Americans (cf. Iceland, Weinberg, \& Steinmetz, 2002) and may have a better appreciation of the diversity of White women.

Finally, Latinas/os' stereotypes were the most difficult to interpret within the theoretical frameworks. It appears that their level of stereotype recognition generally falls somewhere between that of Asian Americans and African Americans. They were more likely to recognize stereotypes of White women as racist (Study 1) and independent and rich (Study 2). To overinterpret these two findings would be unwise, but they suggest the possibility that Latinas/os may perceive White women as having more power than do the other two groups. Perhaps Latinas/os have fewer opportunities for equal status interactions with White women than African Americans or Asian Americans-leading to differences in recognition of stereotypes about White women and power. This is a topic that future researchers may wish to explore.

\section{Gender differences in perceptions of White women}

We also found consistent gender differences in the perceptions of White women in Study 2, indicating that women of all ethnicities were more familiar with and more willing to acknowledge these stereotypes than men were. This is a departure from earlier research that did not demonstrate gender differences in perceptions of White men (Conley et al., 2010). Perhaps women of color are more acutely aware of images of White women because dominant U.S. society measures their appearance against the standard of White women in the media. Even if they do not espouse this standard of beauty personally or in their own culture, many may recognize that they fall short of this societal-level standard of beauty. (Of course, the vast majority-some would argue all_ of White women themselves fall short of these ideals as well.)

\section{Limitations and future directions}

The study of stereotypes of dominant groups is very new, and hence much is left to be done. Below, we suggest a few ways how this research can be extended and improved.

First, the current studies did not provide the best test of the self-categorization perspective. It is possible that people of color use their own group as a baseline from which they generate stereotypes. Self-categorization perspective could be tested more effectively if we asked participants to generate stereotypes about their own group; this would allow us to determine more precisely if those stereotypes are opposite of their stereotypes about White women.

Second, in this study we used two slightly different sets of instructions across studies. In Study 1, we asked participants how others in general perceive White women, whereas in Study 2 we asked participants how members of their own ethnic group would perceive White women. Because the stereotypes were similar across the two studies, we do not believe that the change affected the content of the stereotypes. However, it is possible that the ratings of stereotypes were stronger based on the ethnic group that the participants were referencing, if they were not referencing their own ethnic group in Study 1. Further research should investigate the impact of reference group on stereotype content.

Finally, it would be useful to replicate these results with a representative sample. Likewise, different age groups (e.g., older adults, high school students) might have different stereotypes and, of course, we did not examine all ethnic groups. Moreover, although we did not find differences between specific Asian or Latina/o subgroups (e.g., Mexican Americans vs. Cuban Americans), we also did not have large samples of most of these subgroups. Therefore, the stereotypes of these subgroups may be less uniform than the current data would suggest.

\section{Contribution to stereotyping research}

The current research also provides general information to researchers examining stereotypes. Some researchers have argued that physical descriptors should not be considered stereotypes, whereas others argue for a more general approach to stereotypes as any trait (physical or otherwise) associated with a category of people (cf. Fiske, 1998; Niemann et al., 1994; Worchel \& Rothgerber, 1997). Our research argues for the latter interpretation of stereotypes. That is, the fact that women are stereotyped in terms of their physical characteristics is instructive-it shows that people think of women in physical terms. This may have gone unnoticed by stereotyping researchers because stereotypes of women and men are so often grouped together. However, the fact that White women, but not White men, are defined in terms of their physical appearance gives us important information about the role that gender plays in stereotypes - a role that 
may be occluded by the examination of ethnic groups independent of gender.

Additionally, one stereotype that emerged does not fit neatly with any of the theoretical perspectives we considered: the stereotype dates interracially. This item was more highly ranked and better recognized by African Americans in both studies. This item suggests another pathway through which stereotypes develop: dynamics that represent a point of intraor inter-ethnic group tension (such as historical and contemporary relationships between Black men and White women; Persaud, 2007) may be played out in stereotypes about the groups involved.

\section{Conclusions}

White women often perceive themselves as ethnically "generic" (Frankenburg, 1993). Our research clearly suggests that people of color do not share this perception, but indeed recognize a specific and not entirely flattering image of White women. Thus, we argue that they are ethnically marked. We hope that this research promotes an understanding of the ways in which the intersection of gender and Whiteness may influence the course of relationships between groups in our society.

\section{References}

Backstrom, C. H., \& Hursh-Cesar, G. (1981). Survey research. New York: John Wiley, Inc.

Brekhus, W. (1998). A sociology of the unmarked: Redirecting our focus. Sociological Theory, 16, 34-51.

Conley, T. D., Calhoun, C., Evett, S. R., \& Devine, P. G. (2001). Mistakes that heterosexual people make when trying to appear non-prejudiced: The view from gay people. Journal of Homosexuality, 42, 21-43.

Conley, T. D., Rabinowitz, J. L., \& Rabow, J. (2010). Gordon Gekkos, frat boys and nice guys: The content, dimensions, and structural determinants of multiple ethnic minority groups' stereotypes about White men. Analyses of Social Issues and Public Policy, 10, 69-96.

Conley, T. D., \& Ramsey, L. R. (2011). Killing us softly? Investigating portrayals of women and men in contemporary magazine advertisements. Psychology of Women Quarterly, 35(3), 469-478.

Cowan, G., \& Warren, L. W. (1994). Codependency and gender-stereotyped traits. Psychology of Women Quarterly, 22, 631645.

Cuddy, A. J. C., Fiske, S. T., \& Glick, P. (2007). The BIAS map: Behaviors from intergroup affect and stereotypes. Journal of Personality and Social Psychology, 92, 631-648.

Damarin, S. K. (2000). The mathematically able as a marked category. Gender and Education, 12, 69-85.

Davis, D. M. (1990). Portrayals of women in prime-time network television: Some demographic characteristics. Sex Roles, 23, 325-332.

Downs, A. C., \& Harrison, S. K. (1985). Embarrassing age spots or just plain ugly? Physical attractiveness stereotyping as an instrument of sexism on American television commercials. Sex Roles, 13, 9-19.

Elasmar, M., Hasegawa, K., \& Brain, M. (1999). The portrayal of women in US prime-time television. Journal of Broadcasting and Electronic Media, 43, 20-34.

Evans, E. D., Rutberg, J., Sather, C., \& Turner, C. (1991). Content analysis of contemporary teen magazines for adolescent females. Youth and Society, 23, 99-120.

Fiske, S. T. (1998). Stereotyping, prejudice, and discrimination. In D. T. Gilbert, S. T. Fiske, \& G. Lindzey (Eds.), The handbook of social psychology (Vols. 1 and 2, 4th ed., pp. 357-411). New York: McGraw-Hill.

Fiske, S. T., Cuddy, A. C., Glick, P., \& Xu, J. (2002). A model of (often mixed) stereotype content: Competence and warmth respectively follow from perceived status and competition. Journal of Personality and Social Psychology, 82(6), 878-902.

Frankenburg, R. (1993). White women, race matters. Minneapolis, MN: University of Minnesota Press.

Fredrickson, B., \& Roberts, T. (1997). Objectification theory: Toward understanding women's lived experiences and mental health risks. Psychology of Women Quarterly, 21, 173-206.

Goffman, E. (1963). Stigma: Notes on the management of spoiled identity. Englewood Cliffs, NJ: Prentice-Hall.

Gordijn, E. H., Koomen, W., \& Stapel, D. A. (2001). Level of prejudice in relation to knowledge of cultural stereotypes. Journal of Experimental Social Psychology, 37, 150-157.

Grabe, S., Ward, L. M., \& Hyde, J. S. (2008). The role of the media in body image concerns among women: A meta-analysis of experimental and correlational studies. Psychological Bulletin, 134, 460-476.

Iceland, J., Weinberg, D. H., \& Steinmetz, E. (2002). Racial and ethnic residential segregation in the United States: 1980-2000. U.S. Census Bureau, Census Special Report, CENSR-3, Washington, DC: U.S. Government Printing Office.

Jost, J. T., \& Banaji, M. R. (1994). The role of stereotyping in system justification and the production of false consciousness. British Journal of Social Psychology, 33, 1-27.

Krueger, J. (1996). Personal beliefs and cultural stereotypes about racial characteristics. Journal of Personality and Social Psychology, 71, 536-548.

Landrine, H. (1985). Race x class stereotypes of women. Sex Roles, 13, 6575 .

Makkar, J. K., \& Strube, M. J. (1995). Black women's self-perceptions of attractiveness following exposure to White versus Black beauty standards: The moderating role of racial identity and self-esteem. Journal of Applied Social Psychology, 25, 1547-1566.

Malkin, A. R., Wornian, K., \& Chrisler, J. C. (1999). Content analysis of gendered message on magazine covers. Sex Roles, 40, 647-655.

Mlicki, P. P., \& Ellemers, N. (1998). Being different or being better? National stereotypes and identifications of Polish and 
Dutch students. European Journal of Social Psychology, 26, 97-114.

Niemann, Y. F., Jennings, L., Rozelle, R. M., Baxter, J. C., \& Sullivan, E. (1994). Use of free responses and cluster analysis to determine stereotypes of eight groups. Personality and Social Psychology Bulletin, 20, 379-390.

Norenzayan, A., \& Nisbett, R. (2000). Culture and causal cognition. Current Directions in Psychological Science, 9, 132-135.

Peirce, K. (1993). Socialization of teenage girls through teen-magazine fiction: The making of a new woman or an old lady? Sex Roles, 29, 59-68.

Persaud, R. (2007). Why Black men love White women: Going beyond sexual politics to the heart of the matter. New York: Simon and Schuster.

Prentice, D. A., \& Carranza, E. (2002). What women and men should be, shouldn't be, are allowed to be, and don't have to be: The contents of prescriptive gender stereotypes. Psychology of Women Quarterly, 26, 269-281.

Rich, M. K., \& Cash, T. F. (1993). The American image of beauty: Media representations of hair color for four decades. Sex Roles, 29, 113-124.
Russo, N. F., Feller, L., \& DeLeon, P. H. (1982). Sex role stereotypes in television advertising: Strategies for change in the '80s. Academic Psychology Bulletin, 4, 117-135.

Seidman, S. A. (1992). An investigation of sex-role stereotyping in music videos. Journal of Broadcasting and Electronic Media, 36, 209-216.

Shelton, J. N. (2000). A reconceptualization of how we study issues of racial prejudice. Personality and Social Psychology Review, 4,374-390.

Shelton, J. N., \& Richeson, J. A. (2006). Ethnic minorities' racial attitudes and contact experiences with white people. Cultural Diversity and Ethnic Minority Psychology, 12, 149-164.

Sidanius, J., \& Pratto, F. (1999). Social dominance: An intergroup theory of social hierarchy and oppression. Cambridge, England: Cambridge University.

Signorielli, N., McLeod, D., \& Healy, E. (1994). Gender stereotypes in MTV commercials: The beat goes on. Journal of Broadcasting and Electronic Media, 38, 91-101.

Snow, J. T., \& Harris, M. B. (1986). An analysis of weight and diet content in five women's interest magazines. Journal of
Obesity and Weight Regulation, 5, 194214.

Steele, C. M. (1997). A threat in the air: How stereotypes shape intellectual identity and performance. American Psychologist, 52, 613-629.

Sue, D. W. (2004). Whiteness and ethnocentric monoculturalism: Making the "invisible" visible. American Psychologist, 59, 761-769.

Twenge, J. M., \& Zucker, A. N. (1999). What is a feminist? Evaluations and stereotypes in closed- and open-ended responses. Psychology of Women Quarterly, 23, 591605.

West, C. M. (2008). Mammy, Jezebel, Sapphire, and their homegirls: Developing an "oppositional" gaze toward the images of Black women. In J. C. Chrisler, C. Golden, \& P. D. Rozee (Eds.), Lectures on the psychology of women (4th ed., pp. 287-299). New York: McGraw-Hill.

Worchel, S., \& Rothgerber, H. (1997). Changing the stereotype of the stereotype. In R. Spears, P. J. Oakes, N. Ellemers, \& S. A. Haslam (Eds.), The social psychology of stereotyping and group life (pp. 72-93). Malden, MA: Blackwell Publishing. 